\title{
Inverse Source Problem for a Multiterm Time-Fractional Diffusion Equation with Nonhomogeneous Boundary Condition
}

\author{
L. L. Sun ${ }^{1}{ }^{1}$ and X. B. Yan ${ }^{2}$ \\ ${ }^{1}$ School of Mathematics and Statistics, Northwest Normal University, Lanzhou 730070, China \\ ${ }^{2}$ School of Mathematics and Statistics, Lanzhou University, Gansu 730000, China \\ Correspondence should be addressed to L. L. Sun; sunll0321@163.com
}

Received 7 July 2020; Revised 29 August 2020; Accepted 2 September 2020; Published 19 September 2020

Academic Editor: Nicola L. Rizzi

Copyright (c) 2020 L. L. Sun and X. B. Yan. This is an open access article distributed under the Creative Commons Attribution License, which permits unrestricted use, distribution, and reproduction in any medium, provided the original work is properly cited.

\begin{abstract}
This paper is devoted to identify a space-dependent source function in a multiterm time-fractional diffusion equation with nonhomogeneous boundary condition from a part of noisy boundary data. The well-posedness of a weak solution for the corresponding direct problem is proved by the variational method. We firstly investigate the uniqueness of an inverse initial problem by the analytic continuation technique and the Laplace transformation. Then, the uniqueness of the inverse source problem is derived by employing the fractional Duhamel principle. The inverse problem is solved by the Levenberg-Marquardt regularization method, and an approximate source function is found. Numerical examples are provided to show the effectiveness of the proposed method in one- and two-dimensional cases.
\end{abstract}

\section{Introduction}

It is well known that the standard diffusion equation has been used to describe the Gaussian process of particle motion. Time-fractional diffusion equations (TFDEs in short) are deduced by replacing the standard time derivative with a time-fractional derivative and can be used to describe anomalous diffusion phenomena. Anomalous diffusion deviates from the standard Fickian description of Brownian motion, the main character of which is that its mean squared displacement is a nonlinear growth with respect to time, such as $\left\langle x^{2}(t)\right\rangle \sim t^{\alpha}$. However, as the research problems become more and more complex, the differential order of a timefractional diffusion equation is no longer limited to a fixed number but distributed over the unit interval. This creates TFDEs of distributed order of which a particular case is the multiterm time-fractional diffusion equations (MTFDEs in short).

Direct problems, i.e., initial value problems and initial boundary value problems for MTFDEs, have attracted much more attentions in recent years. For example, the maximum principle in $[1,2]$ and the well-posedness and long-time asymptotic behavior in [3] for general multiterm timefractional diffusion equations are investigated. The numerical solutions are shown in $[4,5]$ by the high-order space-time spectral method and the Galerkin finite element method.

However, in most cases, the parameters that characterize the diffusion process cannot be measured directly or easily. The inverse source problem of diffusion process is aimed at detecting the source function of a physical field from some indirect measurements (such as final time information or boundary measurement) and is of great importance in engineering. There are many ripe regularization theories on the inverse source problems at present, e.g., the reproducing kernel Hilbert space method [6], the Fourier truncation method [7], the Tikhonov regularization method [8], and the modified quasiboundary value method and quasireversibility method $[9,10]$.

In this paper, we investigate an inverse space-dependent source problem in a multiterm time-fractional diffusion equation with nonhomogeneous boundary condition. Let $\Omega$ be a bounded domain in $\mathbb{R}^{d}$ with sufficient smooth boundary $\partial \Omega$. For a fixed positive integer $m$, let $q_{j}$ and $\alpha=\left\{\alpha_{j}: j=1\right.$, $2, \cdots, m\}$ be the positive constants such that $0<\alpha_{m}<\cdots<\alpha_{1}$ 
$<1$. Consider the following initial boundary value problem (IBVP) for a time-fractional diffusion equation

$$
\left\{\begin{array}{l}
\sum_{j=1}^{m} q_{j} \partial_{0+}^{\alpha_{j}} u(x, t)-\Delta u(x, t)=f(x) r(t), \quad(x, t) \in \Omega_{T}:=\Omega \times I, \\
u(x, 0)=\phi(x), x \in \bar{\Omega}, \\
\frac{\partial u}{\partial n}(x, t)=b(x, t), \quad(x, t) \in \partial \Omega \times I
\end{array}\right.
$$

where $I=(0, T)$ and $n$ is the unitary outer normal vector of $\partial \Omega$. We can assume $q_{1}=1$ without loss of generality.

Here, $\partial_{0+}^{\alpha_{j}}$ denotes the Caputo derivative defined by

$$
\partial_{0+}^{\alpha_{j}} u(x, t)=\frac{1}{\Gamma\left(1-\alpha_{j}\right)} \int_{0}^{t} \frac{\partial u(x, s)}{\partial s} \frac{d s}{(t-s)^{\alpha_{j}}}, \quad t>0
$$

where $\Gamma(\cdot)$ is the Gamma function.

If all functions $f(x), r(t), \phi(x)$, and $b(x, t)$, and the parameters $m, q_{j}$ and $\alpha_{j}$ are given appropriately, problem (1) is a direct problem. The inverse problem here is to determine the source term $f(x)$ based on problem (1) and additional data

$$
\left.u(x, t)\right|_{\Gamma}=h(x, t), \quad t \in I
$$

where $\Gamma$ is a nonempty open part of $\partial \Omega$.

To our best knowledge, it has made significant progress in determining the source problem for (1) with part of boundary data. For a single term (i.e. $m=1$ ), Wei et al. identified a time-dependent source term in a multidimensional TFDE from boundary Cauchy data. Zhang et al. [11] proved a uniqueness result to inverse the space-dependent source term in one-dimensional case by using one-point Cauchy data and provided an efficient numerical method. For a general area of high dimension, Wei et al. [12] proved the uniqueness of inverse space-dependent source but no numerical computation. Yan et al. [13] studied an inverse spatialdependent source problem by noisy boundary data in a time-fractional diffusion-wave equation and carried out the numerical inversion experiments by a nonstationary iterative Tikhonov regularization method. For a multiterm case, Jiang et al. [14] established a weak unique continuation property for time-fractional diffusion-advection equations and studied an inverse problem on determining the spatial component in the source term by interior measurements. Very recently, Li et al. [15] investigated an inverse time-dependent source term problem in a MTFDE from the boundary Cauchy data and employed the conjugate gradient method to find the approximate source term.

Nevertheless, as far as the authors know, the most of the existing literature only treated the inverse source problems with homogeneous boundary condition, in which the series expression provides convenience for the argument. The case of nonhomogeneous boundary is also significant from the practical point of view but difficult whether the forward problem or the inverse problem. Although the weak unique continuation property in [14] is also valid for the boundary measurements only taking a zero extension, however, it does not hold true for the nonhomogeneous boundary case. So far, there is no publication on inversion space-dependent source term by the Cauchy data in a MTFDE with nonhomogeneous boundary condition.

Generally speaking, the problems of recovering spatial information from data along a time trace are notoriously severely ill posed. In this paper, we focus on an inverse space-dependent source problem of (1) from the Cauchy data in a general domain. This is an extension and improvement of [12], which dealt with an inverse space-dependent source problem by the boundary measured data in an infinite time interval for a single-term equation with homogeneous boundary. In this study, however, only part of the boundary Cauchy data in a finite time interval $t \in(0, T)(T<\infty)$ is enough to obtain the uniqueness of inverse problem. We firstly obtain the well-posedness for the direct problem by the variational method for studying the inverse problem. For inverse problem, roughly speaking, we first transfer the original forward problems into three IBVPs by the superposition principle and turn the inverse source problem into an inverse initial problem by employing the fractional Duhamel principle. Then, we prove the uniqueness of the inverse initial problem by the analytic continuation technique and the Laplace transformation. Finally, we employ the LevenbergMarquardt regularization method to solve numerically the inverse source problem. Here, we point out that the Levenberg-Marquardt method is modified to a more efficient algorithm without bringing in a differential step in the present paper because of the linearity of the operator. The numerical results for three examples in one- and two-dimensional cases are provided, and the numerical implementation shows the effectiveness and robustness of the proposed methods.

The main result in this paper is the following uniqueness result for the inverse space-dependent source problem.

Theorem 1. Assume that $f \in \mathscr{D}\left((-\Delta+1)^{\gamma}\right)$ for $\gamma>\max \{0$, $d / 2-1\}$ and $r \in C^{1}[0, T]$ with $r(0) \neq 0$, the initial function $\phi$ $\in H^{2}(\Omega)$, and the boundary function $b \in L^{2}(\partial \Omega \times(0, T))$. Suppose $u_{i}(x, t),(i=1,2)$ is the solutions of (1) with $f=f_{i}$, then $u_{1}(x, t)=u_{2}(x, t)$ for $(x, t) \in \Gamma \times I$ implies $f_{1}(x)=f_{2}(x)$.

The remainder of this paper is organized as follows. Some preliminaries are presented in Section 2. The well-posedness for the direct problem is proved in Section 3. In Section 4, we present the uniqueness result of inverse space-dependent source problem. In Section 5, we use the LevenbergMarquardt regularization method to find the approximate space-dependent source function. Numerical results for three examples are provided to illustrate the efficiency of our method in Section 6. Finally, we give a brief conclusion in Section 7.

\section{Preliminaries}

We firstly introduce some preliminaries as follows in this section. 
Definition 2 (see $[16,17])$. If $f(t) \in L^{1}(0, T)$, then for $\alpha>0$, the Riemann-Liouville fractional left-sided integral $I_{0+}^{\alpha} f$ and right-sided integral $I_{T-}^{\alpha} f$ are defined by

$$
\begin{array}{cc}
I_{0+}^{\alpha} f(t)=\frac{1}{\Gamma(\alpha)} \int_{0}^{t} \frac{f(s) d s}{(t-s)^{1-\alpha}}, & 0<t \leq T, \\
I_{T-}^{\alpha} f(t)=\frac{1}{\Gamma(\alpha)} \int_{t}^{T} \frac{f(s) d s}{(s-t)^{1-\alpha}}, & 0 \leq t<T .
\end{array}
$$

Definition 3. (see $[16,17])$. Let $z(t) \in A C(0, T)$, then for $0<$ $\alpha<1$, the Riemann-Liouville fractional left-sided derivative $D_{0+}^{\alpha} y(t)$ and right-sided derivative $D_{T-}^{\alpha} y(t)$ of order $\alpha$ are defined by

$$
\begin{array}{ll}
D_{0+}^{\alpha} y(t)=\frac{1}{\Gamma(1-\alpha)} \frac{d}{d t} \int_{0}^{t} \frac{y(s) d s}{(t-s)^{\alpha}}=: \frac{d}{d t}\left(I_{0+}^{1-\alpha} y\right)(t), \quad 0<t \leq T, \\
D_{T-}^{\alpha} y(t)=-\frac{1}{\Gamma(1-\alpha)} \frac{d}{d t} \int_{t}^{T} \frac{y(s) d s}{(s-t)^{\alpha}}=:-\frac{d}{d t}\left(I_{T-}^{1-\alpha} y\right)(t), & 0 \leq t<T .
\end{array}
$$

Definition 4 (see $[16,18])$. The multinomial Mittag-Leffler function is defined by

$$
E_{\left(\theta_{1}, \cdots, \theta_{m}\right), \theta_{0}}\left(z_{1}, \cdots, z_{m}\right):=\sum_{k=0}^{\infty} \sum_{k_{1}+\cdots+k_{m}=k} \frac{\left(k ; k_{1}, \cdots, k_{m}\right) \Pi_{j=1}^{m} z_{j}^{k_{j}}}{\Gamma\left(\theta_{0}+\sum_{j=1}^{m} \theta_{j} k_{j}\right)}
$$

where $\theta_{0}, \theta_{j} \in \mathbb{R}$, and $z_{j} \in \mathbb{C}(j=1, \cdots, m)$, and $\left(k ; k_{1}, \cdots, k_{m}\right)$ denotes the multinomial coefficient

$$
\left(k ; k_{1}, \cdots, k_{m}\right):=\frac{k !}{k_{1} ! \cdots k_{m} !} \text { with } k=\sum_{j=1}^{m} k_{j} .
$$

For later use, we adopt the abbreviation

$E_{\alpha^{\prime}, \beta}^{\left(\lambda_{n}\right)}(t):=E_{\left(\alpha_{1}, \alpha_{1}-\alpha_{2}, \cdots, \alpha_{1}-\alpha_{m}\right), \beta}\left(-\lambda_{n} t^{\alpha_{1}},-q_{2} t^{\alpha_{1}-\alpha_{2}}, \cdots,-q_{m} t^{\alpha_{1}-\alpha_{m}}\right), \quad t>0$.

We have the following three results on the multinomial Mittag-Leffler function [3].

Lemma 5. Let $\theta_{0}>0,0<\theta_{j}<1(j=1, \cdots, m)$ and $z_{j} \in \mathbb{C}(j=1$ $, \cdots, m)$ be fixed.

Then,

$\frac{1}{\Gamma\left(\theta_{0}\right)}+\sum_{j=1}^{m} z_{j} E_{\left(\theta_{1}, \cdots, \theta_{m}\right), \theta_{0}+\theta_{j}}\left(z_{1}, \cdots, z_{m}\right)=E_{\left(\theta_{1}, \cdots, \theta_{m}\right), \theta_{0}}\left(z_{1}, \cdots, z_{m}\right)$.

Lemma 6. Let $\theta_{0}>0,1>\alpha_{1}>\cdots>\alpha_{m}>0$ be given. Assume that $\alpha_{1} \pi / 2<\mu<\alpha_{1} \pi, \mu \leq\left|\arg z_{1}\right| \leq \pi$ and $\pi-\epsilon \leq\left|\arg z_{j}\right|$ $\leq \pi, j=2, \cdots, m$ with $\epsilon$ small enough. Then, there exists a con- stant $C>0$ depending only $\mu, K, \alpha_{j}(j=1, \cdots, m)$ and $\beta$ such that

$$
\left|E_{\left(\alpha_{1}, \alpha_{1}-\alpha_{2}, \cdots, \alpha_{1}-\alpha_{m}\right), \beta}\left(z_{1}, \cdots, z_{m}\right)\right| \leq \frac{C}{1+\left|z_{1}\right|} .
$$

Lemma 7. Let $1>\alpha_{1}>\cdots>\alpha_{m}>0$. Then,

$$
\frac{d}{d t}\left\{t^{\alpha_{1}} E_{\alpha^{\prime}, 1+\alpha_{1}}^{\left(\lambda_{n}\right)}(t)\right\}=t^{\alpha_{1}-1} E_{\alpha^{\prime}, \alpha_{1}}^{\left(\lambda_{n}\right)}(t), \quad t>0 .
$$

Lemma 8. Assume $r \in L^{\infty}(0, T)$ denote

$$
r_{n}(t)=\int_{0}^{t} r(s)(t-s)^{\alpha_{1}-1} E_{\alpha^{\prime}, \alpha_{1}}^{\left(\lambda_{n}\right)}(t-s) d s, t \in(0, T], n=1,2, \cdots,
$$

then $r_{n}(t) \in C[0, T]$ and $r_{n}(0)=0$.

Proof. Let $e_{n}(t)=t^{\alpha_{1}-1} E_{\alpha^{\prime}, \alpha_{1}}^{\left(\lambda_{n}\right)}(t)$. Then, $r_{n}(t)=r * e_{n}(t)$. By Lemma 6, we have

$$
\left\|e_{n}\right\|_{L^{1}(0, T)}=\int_{0}^{T}\left|t^{\alpha_{1}-1} E_{\alpha^{\prime}, \alpha_{1}}^{\left(\lambda_{n}\right)}(t)\right| d t \leq C \int_{0}^{T} t^{\alpha_{1}-1} d t<\infty .
$$

Therefore, we arrive at $e_{n} \in L^{1}(0, T)$.

Using the Young theorem, we obtain $r_{n}(t) \in C[0, T]$. Combining Lemma 7, we have

$$
\left|r_{n}(0)\right|=\lim _{t \rightarrow 0} r_{n}(t) \leq \lim _{t \rightarrow 0}\|r\|_{L^{\infty}(0, t)} t^{\alpha_{1}} E_{\boldsymbol{\alpha}^{\prime}, 1+\alpha_{1}}^{\left(\lambda_{n}\right)}(t), \quad \forall t \in[0, T] .
$$

Thus, we have $r_{n}(0)=0$.

Lemma 9. Denote $a_{n}(t)=1-\lambda_{n} t^{\alpha_{1}} E_{\boldsymbol{\alpha}^{\prime}, 1+\alpha_{1}}^{\left(\lambda_{n}\right)}(t), \quad t \in(0,+\infty)$, $n=1,2, \cdots$, then

$$
\mathscr{L}\left(a_{n}\right)(s)=\frac{\sum_{j=1}^{m} q_{j} s^{\alpha_{j}-1}}{\sum_{j=1}^{m} q_{j} s^{\alpha_{j}}+\lambda_{n}}, \quad s \in \mathbb{C} \text { with Re } s>0,
$$

where $\mathscr{L}\left(a_{n}\right)$ denotes the Laplace transform of function $a_{n}(t)$.

Proof. First, we can take the Laplace transforms termwise for $\int_{0}^{\infty} e^{-z t} \lambda_{n} t^{\alpha_{1}} E_{\alpha^{\prime}, 1+\alpha_{1}}^{\left(\lambda_{n}\right)}(t) d t$ with Re $z>\lambda_{n}^{1 / \alpha_{1}}$ by its definition (8). Since $\lambda_{n} t^{\alpha_{1}} E_{\alpha^{\prime}, 1+\alpha_{1}}^{\left(\lambda_{n}\right)}(t) \leq C$ by Lemma 6 , we see that $\int_{0}^{\infty}$ $e^{-z t} \lambda_{n} t^{\alpha_{1}} E_{\alpha^{\prime}, 1+\alpha_{1}}^{\left(\lambda_{n}\right)}(t)$ is analytic with respect to $z$ in $\operatorname{Re} z>0$. Therefore, the analytic continuation can be done for Re $z$ $>0$. By Theorem 4.1 in [18], $a_{n}(t)$ satisfies an initial value 
problem for a fractional ODE

$$
\left\{\begin{array}{l}
\sum_{j=1}^{m} q_{j} \partial_{0+}^{\alpha_{j}} a_{n}(t)+\lambda_{n} a_{n}(t)=0, \quad t>0 \\
a_{n}(0)=1
\end{array}\right.
$$
mula

Taking the Laplace transform to (16) and using the for-

$$
\mathscr{L}\left(\partial_{0+}^{\alpha} f\right)(s)=s^{\alpha} \mathscr{L}(f)(s)=s^{\alpha-1} f(0+)
$$

we obtain

$$
\mathscr{L}\left(a_{n}\right)(s)=\frac{\sum_{j=1}^{m} q_{j} s^{\alpha_{j}-1}}{\sum_{j=1}^{m} q_{j} s^{\alpha_{j}}+\lambda_{n}}
$$

\section{Existence and Uniqueness of a Weak Solution for the Direct Problem}

In this section, we will obtain the existence and uniqueness of a weak solution for direct problem (1). First, we define a Hilbert scale space $D\left((-\Delta+1)^{\gamma}\right)$ in $L^{2}(\Omega)$. Denote the eigenvalues of $-\Delta$ with homogeneous Neumann boundary condition as $\lambda_{n}$ and the corresponding eigenfunctions as $\varphi_{n}$ $\in\left\{\psi \in H^{2}(\Omega) ; \partial \psi /\left.\partial n\right|_{\partial \Omega}=0\right\}$ such that $\Delta \varphi_{n}=-\lambda_{n} \varphi_{n}$.

We know that $0=\lambda_{1}<\lambda_{2} \leq \lambda_{3} \leq \cdots \leq \lambda_{n} \leq \cdots$ counting according to the multiplicities, and $\left\{\varphi_{n}\right\}$ is an orthonormal basis in $L^{2}(\Omega)$. Define the Hilbert scale space

$$
\mathscr{D}\left((-\Delta+1)^{\gamma}\right)=\left\{\psi \in L^{2}(\Omega) ; \sum_{n=1}^{\infty}\left(\lambda_{n}+1\right)^{2 \gamma}\left|\left(\psi, \varphi_{n}\right)\right|^{2}<\infty\right\},
$$

where $(\cdot, \cdot)$ is the inner product in $L^{2}(\Omega)$ and define its norm

$$
\|\psi\|_{\mathscr{D}\left((-\Delta+1)^{\gamma}\right)}=\left(\sum_{n=1}^{\infty}\left(\lambda_{n}+1\right)^{2 \gamma}\left|\left(\psi, \varphi_{n}\right)\right|^{2}\right)^{1 / 2}
$$

Here, one points out that the number 1 in the operator $-\Delta+1$ is not necessary. It may be any nonzero positive number.

In the following, we assume that the initial data $\phi(x) \epsilon$ $H^{2}(\Omega)$, the boundary data $b(x, t) \epsilon L^{2}(\partial \Omega \times I)$, and the source functions, $f(x) \epsilon L^{2}(\Omega) r(t) \in L^{2}(0, T)$. We give a weak formulation for direct problem (1) and prove that its solution exists uniquely.
Let $u(x, t)$ be the smooth solution of (1). Denote $v(x, t)$ $=u(x, t)-\phi(x)$, then $v$ solves the following IBVP

$$
\left\{\begin{array}{l}
\sum_{j=1}^{m} q_{j} \partial_{0+}^{\alpha_{j}} v(x, t)-\Delta v(x, t)=\bar{f}(x, t), \quad(x, t) \in \Omega_{T}, \\
v(x, 0)=0, \quad x \in \bar{\Omega}, \\
\frac{\partial v}{\partial n}(x, t)=b-\frac{\partial \phi}{\partial n}, \quad(x, t) \in \partial \Omega \times I,
\end{array}\right.
$$

where $\bar{f}(x, t)=f(x) r(t)+\Delta \phi(x)$.

Let $H^{\alpha}(0, T)$ be the fractional Sobolev space on the interval $(0, T)$ (see, e.g., Adams [19]).

We set

$$
{ }_{0} H^{a_{1}}(0, T)=\left\{u \in H^{a_{1}}(0, T): u(0)=0\right\},
$$

if $1 / 2<\alpha_{1} \leq 1$ and we identify ${ }_{0} H^{a_{1}}(0, T)$ with $H^{\alpha_{1}}(0, T)$ for $0<\alpha_{1}<1 / 2$. For $0<\alpha_{1}<1$, we define a space

$$
B^{\alpha_{1}}\left(\Omega_{T}\right):={ }_{0} H^{\alpha_{1}}\left(0, T ; L^{2}(\Omega)\right) \cap L^{2}\left(0, T ; H^{1}(\Omega)\right),
$$

equipped with the norm

$$
\|u\|_{B^{\alpha_{1}}\left(\Omega_{T}\right)}=\left\{\|u\|_{H^{\alpha_{1}}\left(0, T ; L^{2}(\Omega)\right)}^{2}+\|u\|_{L^{2}\left(0, T ; H^{1}(\Omega)\right)}^{2}\right\}^{1 / 2} .
$$

Referring to [20], we know that $B^{\alpha_{1}}\left(\Omega_{T}\right)$ is a Hilbert space, and for $\alpha_{1} \in(0,1 / 2)$, we have $\|u\|_{H^{\alpha}\left(0, T ; L^{2}(\Omega)\right)}^{2} \cong$ $\|u\|_{L^{2}\left(0, T ; L^{2}(\Omega)\right)}^{2}+\left\|D_{0+}^{\alpha} u\right\|_{L^{2}\left(0, T ; L^{2}(\Omega)\right)}^{2} \cong\|u\|_{L^{2}\left(0, T ; L^{2}(\Omega)\right)}^{2}+$ $\left\|D_{T-}^{\alpha} u\right\|_{L^{2}\left(0, T ; L^{2}(\Omega)\right)}^{2}$, where $D_{0+}^{\alpha} u$ and $D_{T-}^{\alpha} u$ denote the Riemann-Liouville fractional left derivative and right derivative of order $\alpha$ (see Definition 3). Based on [20-22], we can deduce a weak formulation for problem (21) as follows.

Find $v \in B^{\alpha_{1} / 2}\left(\Omega_{T}\right)$ such that

$$
A(v, w)=F(w), \forall w \in B^{\alpha_{1} / 2}\left(\Omega_{T}\right)
$$

where

$$
\begin{gathered}
A(v, w):=\sum_{j=1}^{m}\left(q_{j} D_{0+}^{\alpha_{j} / 2} v, D_{T-}^{\alpha_{j} / 2} w\right)_{\Omega_{T}}+(\nabla v, \nabla w)_{\Omega_{T}}, \\
F(w):=(\bar{f}, w)_{\Omega_{T}}+\left(b-\frac{\partial \phi}{\partial n}, w\right)_{\partial \Omega \times I} .
\end{gathered}
$$

Now, we could obtain the following theorem.

Theorem 10. Let $f \in L^{2}(\Omega), r(t) \in L^{2}(0, T)$, and $\phi \in H^{2}(\Omega)$. Then, there exists a unique solution $v(x, t)$ to (25) and the solution satisfies 
$\|v\|_{B^{\alpha_{1} / 2}\left(\Omega_{T}\right)} \leq C\left(\alpha_{1}, T, q_{j}\right)\left(\|f\|_{L^{2}(\Omega)}\|r\|_{L^{2}(0, T)}+\|\phi\|_{H^{2}(\Omega)}+\|b\|_{L^{2}(\partial \Omega \times I)}\right)$,

where $C\left(\alpha_{1}, T, q_{j}\right)>0$ is a constant independent of $v$.

Proof. The well-posed of problem (25) is guaranteed by the well-known Lax-Milgram theorem. The continuity of the functional $F$ is obvious. Here, we give the continuity and coercivity of the bilinear form A. By the Cauchy-Schwarz inequality, we have

$$
\begin{aligned}
|A(v, w)| & \leq \sum_{j=1}^{m} q_{j}\left\|D_{0+}^{\alpha_{j} / 2} v\right\|_{L^{2}\left(\Omega_{T}\right)}\left\|D_{T-}^{\alpha_{j} / 2} w\right\|_{L^{2}\left(\Omega_{T}\right)}+\|\nabla v\|_{L^{2}\left(\Omega_{T}\right)}\|\nabla w\|_{L^{2}\left(\Omega_{T}\right)} \\
& \leq C m \max _{1 \leq j \leq m}\left\{q_{j}\right\}\|v\|_{B^{\alpha_{1} / 2}\left(\Omega_{T}\right)}\|w\|_{B^{\alpha_{1} / 2}\left(\Omega_{T}\right)}+\|v\|_{B^{\alpha_{1} / 2}\left(\Omega_{T}\right)}\|w\|_{B^{\alpha_{1} / 2}\left(\Omega_{T}\right)} \\
& \leq\left(1+C m \max _{1 \leq j \leq m}\left\{q_{j}\right\}\right)\|v\|_{B^{\alpha_{1} / 2}\left(\Omega_{T}\right)}\|w\|_{B^{\alpha_{1} / 2}\left(\Omega_{T}\right)} .
\end{aligned}
$$

On the other hand, we arrive at

$$
\begin{aligned}
A(w, w) & \geq \sum_{j=1}^{m} q_{j} \cos \left(\pi \alpha_{j} / 2\right)\left\|D_{0+}^{\alpha_{1} / 2} w\right\|_{L^{2}\left(\Omega_{T}\right)}^{2}+\|\nabla w\|_{L^{2}\left(\Omega_{T}\right)}^{2} \\
& \geq \frac{\cos \left(\pi \alpha_{1} / 2\right)\left\|D_{0+}^{\alpha_{1} / 2} w\right\|_{L^{2}\left(\Omega_{T}\right)}^{2}}{2}+\frac{\cos \left(\pi \alpha_{1} / 2\right)\|w\|_{L^{2}\left(\Omega_{T}\right)}^{2}+\|\nabla w\|_{L^{2}\left(\Omega_{T}\right)}^{2}}{2}\left\|\frac{c \cos \left(\pi \alpha_{1} / 2\right)}{2}\right\| w \|_{B^{\alpha_{1} / 2}\left(\Omega_{T}\right)}^{2},
\end{aligned}
$$

where we use Lemma 2.2 of [20] in the first inequality and the embedding theorem in the second inequality. In order to obtain the stability of (27), we can take $w=v$ in (25) and use (29) to obtain

$$
\|v\|_{B^{\alpha_{1} / 2}\left(\Omega_{T}\right)} \leq \frac{C}{\cos \left(\pi \alpha_{1} / 2\right)}\left(\|f\|_{L^{2}(\Omega)}\|r\|_{L^{2}(0, T)}+\|\phi\|_{H^{2}(\Omega)}+\|b\|_{L^{2}(\partial \Omega \times I)}\right) .
$$

\section{Uniqueness for the Inverse Problem}

In order to prove our main result, we firstly introduce the following famous fractional Duhamel principle.

Assume $u$ solves equation (1) with $\phi=0$ and $b=0$, and let $v$ be the solution of the following problem

$$
\left\{\begin{array}{l}
\sum_{j=1}^{m} q_{j} \partial_{0+}^{\alpha_{j}} v(x, t)-\Delta v(x, t)=0, \quad(x, t) \in \Omega_{T}, \\
v(x, 0)=f(x), \quad x \in \bar{\Omega}, \\
\frac{\partial v}{\partial n}(x, t)=0, \quad(x, t) \in \partial \Omega \times I .
\end{array}\right.
$$

Lemma 11. (see Lemma 4.2 of [2]). Let $u$ be the solution to (1) with $\phi=0$ and $b=0$, where $r \in C^{1}[0, T]$ and $f \in \mathscr{D}\left((-\Delta+1)^{\epsilon}\right)$ with some $\epsilon>0$. Then, $u$ allows the representation

$$
u(\cdot, t)=\int_{0}^{t} \mu(t-s) v_{f}(\cdot, s) d s, 0<t \leq T,
$$

where $v_{f}$ solves homogeneous problem (31) with $f$ as the initial data, and $\mu$ satisfies

$$
\sum_{j=1}^{m} q_{j} I_{0+}^{1-\alpha_{j}} \mu(t)=r(t), \quad 0<t \leq T .
$$

Moreover, there exists a unique $\mu \in L^{1}(0, T)$ satisfying (33), and there is a constant $C>0$ independent of $t$ such that

$$
|\mu(t)| \leq C t^{\alpha_{1}-1} \text { a.e.t } \in(0, T) .
$$

Based on the method of separation of variables, we could obtain a formal solution to the homogeneous boundary problem of (1) given by

$$
u(x, t)=\sum_{n=1}^{\infty} a_{n}(t) \phi_{n} \varphi_{n}(x)+\sum_{n=1}^{\infty} r_{n}(t) f_{n} \varphi_{n}(x),
$$

where $a_{n}(t)=\left(1-\lambda_{n} t^{\alpha_{1}} E_{\alpha^{\prime}, 1+\alpha_{1}}^{\left(\lambda_{n}\right)}(t)\right), r_{n}(t)=\int_{0}^{t} r(s)(t-s)^{\alpha_{1}-1}$ $E_{\alpha^{\prime}, 1+\alpha_{1}}^{\left(\lambda_{n}\right)}(t-s) d s, \phi_{n}=\left(\phi, \varphi_{n}\right)$ and $f_{n}=\left(f, \varphi_{n}\right)$.

Now, we proceed to the proof of the uniqueness of the inverse initial problem for the homogeneous initial-boundary value problem (31).

Theorem 12. Assume that $f$ and $\tilde{f} \in \mathscr{D}\left((-\Delta+1)^{\gamma}\right)$ for $\gamma>$ $\max \{0, d / 2-1\}$. Suppose $v(x, t)$ and $\tilde{v}(x, t)$ are the weak solutions of (31) with respect to initial conditions $f$ and $\tilde{f}$, respectively, then $v(x, t)=\tilde{v}(x, t)$ for $(x, t) \in \Gamma \times I$ implies $f$ $(x)=\tilde{f}(x)$ in $L^{2}(\Omega)$.

Proof. By (35), the expressions $v(x, t)$ and $\tilde{v}(x, t)$ are given by

$$
v(x, t)=\sum_{n=1}^{\infty} a_{n}(t) f_{n} \varphi_{n}(x), \quad \tilde{v}(x, t)=\sum_{n=1}^{\infty} a_{n}(t) \tilde{f}_{n} \varphi_{n}(x),
$$

where $\tilde{f}_{n}=\left(\tilde{f}, \varphi_{n}\right)$. Next, we prove the uniform convergence of above series. By the Sobolev embedding theorem, we have

$$
\left\|\varphi_{n}\right\|_{C(\bar{\Omega})} \leq C\left\|\varphi_{n}\right\|_{H^{2 m}(\Omega)},
$$

for $m>d / 4$. Moreover, we have

$$
\left\|\varphi_{n}\right\|_{H^{2 m}(\Omega)} \leq C\left\|(-\Delta+1)^{m} \varphi_{n}\right\|_{L^{2}(\Omega)}=C\left(\lambda_{n}+1\right)^{m} .
$$

On the one hand, when $z \in S_{1}:=\{z \in \mathbb{C} ;|\arg z| \leq \min \{$ $\left.(\pi-\mu) / \alpha_{1}, \epsilon /\left(\alpha_{1}-\alpha_{m}\right), \pi\right\}$, by Lemma 5 and Lemma 6 , combining (37) and (38) and the Cauchy-Schwarz inequality, we arrive at 


$$
\begin{aligned}
& \sum_{n=2}^{\infty} \max _{x \in \bar{\Omega}}\left|\left(1-\lambda_{n} z^{\alpha_{1}} E_{\alpha^{\prime}, 1+\alpha_{1}}^{\left(\lambda_{n}\right)}(z)\right) f_{n} \varphi_{n}(x)\right| \\
& \quad=\sum_{n=2}^{\infty} \max _{x \in \bar{\Omega}}\left|E_{\alpha^{\prime}, 1}^{\left(\lambda_{n}\right)}(z)+\sum_{j=2}^{m} q_{j} z^{\alpha_{1}-\alpha_{j}} E_{\alpha^{\prime}, 1+\alpha_{1}-\alpha_{2}}^{\left(\lambda_{n}\right)}(z)\right|\left|f_{n} \varphi_{n}(x)\right| \\
& \leq C \sum_{n=2}^{\infty} \sum_{j=1}^{m}\left|\frac{z^{\alpha_{1}-\alpha_{j}}}{1+\lambda_{n} z^{\alpha_{1}}}\right|\left\|f_{n} \varphi_{n}(x)\right\|_{L^{\infty}(\bar{\Omega})} \leq C \sum_{n=2}^{\infty}\left|f_{n}\right|\left(\lambda_{n}+1\right)^{m-1} \\
& \quad \leq C\left(\sum_{n=2}^{\infty} \frac{1}{\left(\lambda_{n}+1\right)^{2(\gamma+1-m)}}\right)^{1 / 2}\left(\sum_{n=2}^{\infty}\left(\lambda_{n}+1\right)^{2 \gamma}\left|f_{n}\right|\right)^{1 / 2}
\end{aligned}
$$

By the book [23], we know that $\lambda_{n} \geq C n^{2 / d}, n \in \mathbb{N}$. If we choose $\gamma>m+(d / 4)-1$, i.e., $\gamma>(d / 2)-1$, then the first series of the last term is convergent. By $f \in \mathscr{D}\left((-\Delta+1)^{\gamma}\right)$, we know that the above series is convergent uniformly over $z \in S_{1}$ for $x \in \bar{\Omega}$. On the other hand, we know that the multinomial Mittag-Leffler function $E_{\alpha^{\prime}, 1+\alpha_{1}}^{\left(\lambda_{n}\right)}(z)$ is analytic over the domain $S_{2}:=\{z \in \mathbb{C} ;|z|>0,|\arg z|<\pi\}$ based on its definition. Thus, the expressions $v(x, t)$ and $\tilde{v}(x, t)$ are analytic over the domain $z \in S_{1} \cap S_{2}$ for $x \in \bar{\Omega}$ based on the Weierstrass theorem. Especially, $u(x, t)$ and $v(x, t)$ can be extended to $\bar{\Omega} \times(0, \infty)$.

Therefore, by the analytic continuation, the condition $v$ $(x, t)=\tilde{v}(x, t)$ for $(x, t) \in \Gamma \times I$ gives

$$
\sum_{n=1}^{\infty} a_{n}(t) f_{n} \varphi_{n}(x)=\sum_{n=1}^{\infty} a_{n}(t) \tilde{f}_{n} \varphi_{n}(x), \quad x \in \Gamma, t>0 .
$$

We set $\sigma(-\Delta)=\left\{\mu_{n}\right\}_{n \in \mathbb{N}}$ with $0=\mu_{1}<\mu_{2}<\mu_{3}<\cdots$ and denote by $\left\{\varphi_{n_{k}}\right\} 1 \leq k \leq m_{n}$ an orthonormal basis of $\operatorname{Ker}\left(\mu_{n}\right.$ $+\Delta)$. Now, we consider $\sigma(-\Delta)$ as a set, not as a sequence with multiplicities. Then, we can rewrite (40) by

$$
\sum_{n=1}^{\infty}\left(\sum_{k=1}^{m_{n}} f_{n_{k}} \varphi_{n_{k}}(x)\right) \tilde{a}_{n}(t)=\sum_{n=1}^{\infty}\left(\sum_{k=1}^{m_{n}} \tilde{f}_{n_{k}} \varphi_{n_{k}}(x)\right) \tilde{a}_{n}(t), x \in \Gamma, t>0,
$$

where $\tilde{a}_{n}(t)=\left(1-\mu_{n} t^{\alpha_{1}} E_{\alpha^{\prime}, 1+\alpha_{1}}^{\left(\mu_{n}\right)}(t)\right)$ and $f_{n_{k}}=\left(f, \varphi_{n_{k}}\right)$. From (39), one obtains

$$
\left|e^{-t \operatorname{Re} z} \sum_{n=2}^{\infty} f_{n} a_{n}(t) \varphi_{n}(x)\right| \leq C e^{-t \operatorname{Re} z}\|f\|_{D\left((-\Delta+1)^{\gamma}\right)}
$$

and $e^{-t \text { Re } z}$ is integrable in $t \in(0,+\infty)$ for fixed $z$ satisfying $\operatorname{Re} z>0$. By the Lebesgue convergence theorem, we can take the Laplace transform for (41). By Lemma 9, we have

$$
\mathscr{L}\left(a_{n}\right)(s)=\frac{\sum_{j=1}^{m} q_{j} s^{\alpha_{j}-1}}{\sum_{j=1}^{m} q_{j} s^{\alpha j}+\lambda_{n}}, \operatorname{Re} s>0
$$

Therefore, we arrive at

$$
\begin{aligned}
& \sum_{n=1}^{\infty}\left(\sum_{k=1}^{m_{n}} f_{n_{k}} \varphi_{n_{k}}(x)\right) \frac{\sum_{j=1}^{m} q_{j} s^{\alpha_{j-1}}}{\sum_{j=1}^{m} q_{j} s^{\alpha_{j}}+\mu_{n}} \\
& \quad=\sum_{n=1}^{\infty}\left(\sum_{k=1}^{m_{n}} \tilde{f}_{n_{k}} \varphi_{n_{k}}(x)\right) \frac{\sum_{j=1}^{m} q_{j} s^{\alpha_{j}+1}}{\sum_{j=1}^{m} q_{j} s^{\alpha_{j}}+\mu_{n}}, \quad x \in \Gamma, \operatorname{Re} s>0,
\end{aligned}
$$

which implies

$\sum_{n=1}^{\infty} \sum_{k=1}^{m_{n}} f_{n_{k}} \frac{\varphi_{n_{k}}(x) \eta}{\eta+\mu_{\eta}}=\sum_{n=1}^{\infty} \sum_{k=1}^{m_{n}} \tilde{f}_{n_{k}} \frac{\varphi_{n_{k}}(x) \eta}{\eta+\mu_{n}}, \quad x \in \Gamma, \eta \in S_{3} \supset \mathbb{R}^{+}$,

where $\eta=\sum_{j=1}^{m} q_{j} s^{\alpha_{j}}$. As

$\sum_{n=1}^{\infty} \sum_{k=1}^{m_{n}}\left|\left(f, \varphi_{n_{k}}\right) \frac{\eta}{\eta+\mu_{n}} \varphi_{n_{k}}(x)\right| \leq \sum_{n=1}^{\infty}\left\|\left(f, \varphi_{n}\right) \varphi_{n}\right\|_{L^{\infty}(\bar{\Omega})}\left|\frac{\eta}{\eta+\lambda_{n}}\right|$,

one can see that the above series is internally closed uniform convergence in $\eta \in \mathbb{C} \backslash\left\{-\mu_{n}\right\}_{n=1}^{\infty}$ from (39). Using the Weierstrass theorem, both sides of (45) are analytic in $\eta \in \mathbb{C} \backslash$ $\left\{-\mu_{n}\right\}_{n=1}^{\infty}$. Therefore, one can analytically continue $\eta$ such that (45) holds for $\eta \in \mathbb{C} \backslash\left\{-\mu_{n}\right\}_{n=1}^{\infty}$.

Now, we can take a suitable disk which only includes $\mu_{l}$. Using the Cauchy integral theorem, integrating (45) along this disk, we obtain that $\mu_{l}(x)=\sum_{k=1}^{m_{l}}\left(f-\tilde{f} \varphi_{l_{k}}\right) \varphi_{l_{k}}(x)=0, x$ $\in \Gamma$. Since $\left(\Delta+\mu_{l}\right) u_{l}=0$ in $\Omega$, and $u_{l}=\partial_{u_{l}} / \partial n=0$, on $\Gamma$ the uniqueness of the Cauchy problem for elliptic equations (e.g., see Theorem 3.3.1 of Isakov [24], p. 58) implies $u_{l}=0$ in $\Omega$ for each $l \in \mathbb{N}$. Combining the linearly independence of $\left\{\varphi_{l_{k}}\right\}_{1 \leq k \leq m_{l}}$ in $\Omega$, we obtain that $\left(f-\tilde{f}, \varphi_{l_{k}}\right)=0$ for $1 \leq k$ $\leq m_{l}, l \in \mathbb{N}$. Therefore $f=\tilde{f}$ in $L^{2}(\Omega)$.

Remark 13. Assume that $\phi=0$ and $b=0, f \in \mathscr{D}\left((-\Delta+1)^{\gamma}\right)$ for $\gamma>\max \{0, d / 2-1\}$ and $r \in L^{\infty}(0, \infty)$, then the solution of (1) $u \in C(\bar{\Omega} \times(0,+\infty))$.

Proof. From (35), the solution $u(x, t)$ is given by

$$
u(x, t)=\sum_{n=1}^{\infty} r_{n}(t) f_{n} \varphi_{n}(x)
$$

Let $t_{0}>0$ be arbitrarily fixed. For $t \geq t_{0}$, by Lemma 7 and Lemma 6, we have 


$$
\begin{aligned}
& \sum_{n=2}^{\infty} \max _{x \in \bar{\Omega}}\left|\int_{0}^{t} r(s)(t-s)^{a_{1}-1} E_{\alpha^{\prime}, a_{1}}^{\left(\lambda_{n}\right)}(t-s) d s f_{n} \varphi_{n}(x)\right| \\
& \quad \leq\|r\|_{\infty} \sum_{n=2}^{\infty}\left\|f_{n} \varphi_{n}\right\|_{L^{\infty}(\bar{\Omega})} \frac{C t^{\alpha_{1}}}{1+\lambda_{n} t^{\alpha_{1}}} \leq C\|r\|_{\infty} \sum_{n=2}^{\infty}\left|f_{n}\right|\left(\lambda_{n}+1\right)^{m-1} \\
& \quad \leq C\|r\|_{\infty}\left(\sum_{n=2}^{\infty} \frac{1}{\left(\lambda_{n}+1\right)^{2(\gamma+1-m)}}\right)^{1 / 2}\left(\sum_{n=2}^{\infty}\left(\lambda_{n}+1\right)^{2 \gamma}\left|f_{n}\right|^{2}\right)^{1 / 2} \\
& \quad \leq C\|r\|_{\infty}\|f\|_{\mathscr{D}\left((-\Delta+1)^{\gamma}\right),}
\end{aligned}
$$

where $\gamma>\max \{0, d / 2-1\}$. Thus, the series (48) is also convergent on $\bar{\Omega} \times\left[t_{0}, \infty\right)$ uniformly. By Lemma 8 , we know $u$ in (48) is continuous on $\bar{\Omega} \times[t 0, \infty)$.

Finally, we can proceed to prove the main result Theorem 1 raised at the end of the introduction.

Proof of Theorem 1. As we know, by the principle of linear superposition, we have $u=u_{f}+u_{\phi}+u_{b}$ and they solve the following three IBVPs:

$$
\begin{cases}\sum_{j=1}^{m} q_{j} \partial_{0+}^{\alpha_{j}} u_{f}(x, t)-\Delta_{f}(x, t)=f(x) r(t), & (x, t) \in \Omega_{T}, \\ u_{f}(x, 0)=0, & x \in \bar{\Omega}, \\ \frac{\partial u_{f}}{\partial n_{n}}(x, t)=0, & (x, t) \in \partial \Omega \times I,\end{cases}
$$

$$
\begin{aligned}
& \begin{cases}\sum_{j=1}^{m} q_{j} \partial_{0+}^{\alpha_{j}} u_{\phi}(x, t)-\Delta u_{\phi}(x, t)=0 & (x, t) \in \Omega_{T}, \\
u_{\phi}(x, 0)=\phi(x), & x \in \bar{\Omega}, \\
\frac{\partial u_{\phi}}{\partial n}(x, t)=0, & (x, t) \in \partial \Omega \times I,\end{cases} \\
& \begin{cases}\sum_{j=1}^{m} q_{j} \partial_{0+}^{\alpha_{j}} u_{b}(x, t)-\Delta u_{b}(x, t)=0, & (x, t) \in \Omega_{T} \\
u_{b}(x, 0)=0, & x \in \bar{\Omega}, \\
\frac{\partial u_{b}}{\partial n}(x, t)=b(x, t), & (x, t) \in \partial \Omega \times I .\end{cases}
\end{aligned}
$$

It is not hard to find that the solutions of IBVPs (50) and (51) are independent of the source term $f$, and then, the inverse source problem becomes more precise, i.e., can we determine $f(x)$ from $u_{f}(x, t)$ for $(x, t) \in \Gamma \times I$ uniquely. According to Remark 13, we know that $u_{f} \in C(\bar{\Omega} \times(0, \infty))$. By Lemma 11, we know that $u_{f}(x, t)=0, x \in \Gamma, 0<t \leq T$ deduce

$$
\int_{0}^{t} \mu(t-s) v_{f}(x, s) d s=0, x \in \Gamma, \quad 0<t \leq T .
$$

By the Fibini theorem, we obtain from (33) that

$$
\begin{aligned}
\sum_{j=1}^{m} & q_{j} I_{0+}^{1-\alpha_{j}} \int_{0}^{t} \mu(t-s) v_{f}(x, s) d s \\
& =\sum_{j=1}^{m} \frac{q_{j}}{\Gamma\left(1-\alpha_{j}\right)} \int_{0}^{t} \frac{d \tau}{(t-\tau)^{\alpha_{j}}} \int_{0}^{\tau} \mu(\tau-s) v_{f}(x, s) d s, \\
& =\sum_{j=1}^{m} \frac{q_{j}}{\Gamma\left(1-\alpha_{j}\right)} \int_{0}^{t} v_{f}(x, s) d s \int_{0}^{t} \frac{\mu(\tau-s)}{(t-\tau)^{\alpha_{j}}} d \tau, \\
& =\int_{0}^{t} r(t-s) v_{f}(x, s) d s .
\end{aligned}
$$

So, we have $\int_{0}^{t} r(t-s) v_{f}(x, s) d s=0, x \in \Gamma, 0<t \leq T$. Differentiating the above equality with respect to $t$, we arrive at $r(0) v_{f}(x, t)+\int_{0}^{t} r^{\prime}(t-s) v_{f}(x, s) d s=0$. As the assumption $r(0) \neq 0$, we have by the generalized Minkowski inequality that

$$
\begin{aligned}
\left\|v_{f}(\cdot, t)\right\|_{L^{2}(\Gamma)} & \leq \frac{1}{r(0)} \int_{0}^{t} r^{\prime}(t-s)\left\|v_{f}(\cdot, s)\right\|_{L^{2}(T)} d s \\
& \leq \frac{\|r\|_{C^{1}[0, T]}}{r(0)} \int_{0}^{t}\left\|v_{f}(\cdot, s)\right\|_{L^{2}(T)} d s .
\end{aligned}
$$

Using the Gronwall inequality, we have $v_{f}(x, t)=0, x \in \Gamma$, $0<t \leq T$. Therefore, we know $f(x)=0$ by Theorem 12 . Thus, we complete the proof.

\section{Levenberg-Marquardt Regularization Method}

In this section, we solve numerically the inverse problem of identifying the space-dependent source $f(x)$. As we know, most of inversion algorithms are based on regularization strategies so as to overcome ill-posedness of inverse problems, and different kinds of inverse problems may need different approximate methods on the basis of conditional well-posedness analysis. In this paper, we employ the Levenberg-Marquardt regularization technique to obtain an approximation to the source term.

Based on Theorem 10, we can define a forward linear operator

$$
\mathscr{F}: \quad f(x) \in L^{2}(\Omega) \rightarrow u(x, t ; f) \in L^{2}(\Gamma \times(0, T)) .
$$

Thus, the inverse problem is translated into solving the following abstract operator equation

$$
\mathscr{F} f=\left.u(x, t)\right|_{\Gamma \triangleq} h(x, t), \quad t \in I .
$$

From Theorem 10, we know that $u(x, t ; f) \in B^{\alpha_{1} / 2}\left(\Omega_{T}\right)$. By the trace theorem in [25], we know $\left.u(x, t ; f)\right|_{\Gamma \times I} \in L^{2}(0$, $\left.T ; H^{1 / 2}(\Gamma)\right) \cap_{0} H^{a_{1} / 4}\left(0, T ; L^{2}(\Gamma)\right) \rightarrow L^{2}(\Gamma \times(0, T))$ 
compactly, then the operator $\mathscr{F}: L^{2}(\Omega) \rightarrow L^{2}(\Gamma \times(0, T))$ is compact and thus the inverse source problem is ill-posed. In order to ensure a stable numerical reconstruction of $f(x)$ , we give the following minimization problem with the Tikhonov-type regularization term

$\min _{f \in \mathscr{D}\left((-\Delta+1)^{\gamma}\right)} J_{\mu}(f)=\left\|\mathscr{F} f-h^{\delta}\right\|_{L^{2}(\Gamma \times(0, T))}^{2}+\mu\left\|f-f^{*}\right\|_{\mathscr{D}\left((-\Delta+1)^{\gamma}\right)}^{2}$,

where $\gamma>d / 2-1, \mu>0$ is a regularization parameter, $f^{*} \epsilon$ $\mathscr{D}\left((-\Delta+1)^{\gamma}\right)$ is a suitable guess of $f$, and $h^{\delta}$ is the noisy function of $h$.

Lemma 14. (see [26]). Assume that $E$ is a uniformly convex Banach space. Let $\left(x_{n}\right)$ be a sequence in E such that $x_{n} \rightarrow x$ weakly $\sigma\left(E, E^{\prime}\right)$ and limsup $\left\|x_{n}\right\| \leq\|x\|$. Then, $x_{n} \rightarrow x$ strongly.

By the canonical book [27], we know that with above minimization problem exists a unique stable solution of regularization. Moreover, the convergence result can be obtained in the following theorem.

Theorem 15. Suppose that $\mathscr{F} f=h$ and the noisy data $h^{\delta} \in$ $L^{2}(\Gamma \times(0, T))$ satisfying $\left\|h^{\delta}-h\right\|_{L^{2}(\Gamma \times(0, T))} \leq \delta$ and let $\mu(\delta)$ satisfy $\mu(\delta) \rightarrow 0$ and $\delta^{2} / \mu(\delta) \rightarrow 0$ as $\delta \rightarrow 0$; then, the minimizer $f_{\mu}^{\delta}$ of variational problem (57) is convergent, i.e., $f_{\mu}^{\delta}$ $\rightarrow f$ in $\mathscr{D}\left((-\Delta+1)^{\gamma}\right)$ as $\delta \rightarrow 0$.

Proof. Let $\delta_{k}$ be any sequence such that $\delta_{k} \rightarrow 0$ and denote $\mu_{k}=\mu\left(\delta_{k}\right)$. Since

$$
\begin{aligned}
& \left\|\mathscr{F} f_{\mu_{k}}^{\delta_{k}}-h^{\delta_{k}}\right\|_{L^{2}(\Gamma \times(0, T))}^{2}+\mu_{k}\left\|f_{\mu_{k}}^{\delta_{k}}-f^{*}\right\|_{\mathscr{D}\left((-\Delta+1)^{\gamma}\right)}^{2} \\
& \quad \leq\left\|\mathscr{F} f-h^{\delta_{k}}\right\|_{L^{2}(\Gamma \times(0, T))}^{2}+\mu_{k}\left\|f-f^{*}\right\|_{\mathscr{D}\left((-\Delta+1)^{\gamma}\right)}^{2} \\
& \quad \leq \delta_{k}^{2}-\mu_{k}\left\|f-f^{*}\right\|_{\mathscr{D}\left((-\Delta+1)^{\gamma}\right)}^{2},
\end{aligned}
$$

we have $\left\|f_{\mu_{k}}^{\delta_{k}}-f^{*}\right\|_{D\left((-\Delta+1)^{\gamma}\right)}^{2} \leq \delta_{k}^{2} / \mu_{k}+\left\|f-f^{*}\right\|_{\mathscr{D}\left((-\Delta+1)^{\gamma}\right)}^{2}$ and $\limsup _{\delta_{k} \rightarrow 0}\left\|f_{\mu_{k}}^{\delta_{k}}-f^{*}\right\|_{\mathscr{D}\left((-\Delta+1)^{\gamma}\right)} \leq\left\|f-f^{*}\right\|_{\mathscr{D}\left((-\Delta+1)^{\gamma}\right)}$ by the condition for $\mu$. So we know that $\left\|f_{\mu_{k}}^{\delta_{k}}\right\|_{\mathscr{D}\left((\Delta+1)^{\gamma}\right)}$ is bounded and has a weak convergence subsequence by the reflexivity of $\mathscr{D}$ $\left((-\Delta+1)^{\gamma}\right)$ and also denotes $f_{\mu_{k}}^{\delta_{k}}$ such that $f_{\mu_{k}}^{\delta_{k}} \rightarrow \mathrm{z}$ in $\mathscr{D}$ $\left((-\Delta+1)^{\gamma}\right)$.

Since the linear operator $\mathscr{F}$ is bounded, we know that $\mathscr{F} f_{\mu_{k}}^{\delta_{k}} \rightarrow \mathscr{F}_{z} \quad$ in $L^{2}(\Gamma \times(0, T))$.

From (58), we have $\left\|\mathscr{F} f_{\mu_{k}}^{\delta_{k}}-h^{\delta_{k}}\right\|_{L^{2}(\Gamma \times(0, T))} \leq \delta_{k}^{2}+\mu_{k}$ $\left\|f-f^{*}\right\|_{\mathscr{D}\left((-\Delta+1)^{\gamma}\right)}^{2}$, and thus $\lim _{\delta_{k} \rightarrow 0} f_{\mu_{k}}^{\delta_{k}}=h$ and $\mathscr{F} z=h$. By the uniqueness of $\mathscr{F} f=h$, we know that $z=f$.

By the weak lower semicontinuity of the norm in the Hilbert space, we have $\left\|f-f^{*}\right\|_{\mathscr{D}\left((-\Delta+1)^{\gamma}\right)} \liminf _{\delta_{k} \rightarrow 0}$
$\left\|f_{\mu_{k}}^{\delta_{k}}-f^{*}\right\|_{\mathscr{D}\left((-\Delta+1)^{\gamma}\right)} \leq \limsup _{\delta_{k} \rightarrow 0}\left\|f_{\mu_{k}}^{\delta_{k}}-f^{*}\right\|_{\mathscr{D}\left((-\Delta+1)^{\gamma}\right)} \leq$ $\left\|f-f^{*}\right\|_{\mathscr{D}\left((-\Delta+1)^{\gamma}\right)}$, and hence, we know that $\lim _{\delta_{k} \rightarrow 0}$ $\left\|f_{\mu_{k}}^{\delta_{k}}-f^{*}\right\|_{\mathscr{D}\left((-\Delta+1)^{\gamma}\right)}=\left\|f-f^{*}\right\|_{\mathscr{D}\left((-\Delta+1)^{\gamma}\right)}$. Combing with the weak convergence of $f_{\mu_{k}}^{\delta_{k}}$, we have by Lemma 14 that $f_{\mu_{k}}^{\delta_{k}} \rightarrow$ $f$. From the uniqueness of $\mathscr{F} f=h$, we have that $f_{\mu(\delta)}^{\delta} \rightarrow f$ in $\mathscr{D}\left((-\Delta+1)^{\gamma}\right)$.

In the following, we use the Levenberg-Marquardt method to minimize problem (57). The LevenbergMarquardt method was first introduced by [28, 29], and it is a kind of the Newton-type method. Moreover, it has been well applied to fractional equations, for example, see [3032]. From physical considerations, we know that $h^{\delta}$ is a reasonably close approximation of some ideal $h=\mathscr{F} f$ in the range of $\mathscr{F}$. Let $f^{*}$ be an approximation of $f$. By the linearity expansion for linear operator $\mathscr{F}$ at $f^{*}$, we can obtain

$$
\mathscr{F} f=\mathscr{F} f^{*}+\left(\mathscr{F}_{f}^{\prime} f^{*}\right)\left(f-f^{*}\right) .
$$

Then, the inverse problem $\mathscr{F} f=h^{\delta}$ can be transformed into

$$
\left(\mathscr{F}_{f}^{\prime} f^{*}\right)\left(f-f^{*}\right)=h^{\delta}-\mathscr{F} f^{*}
$$

Therefore, it is easily seen that the problem of minimizing (57) is equivalent to minimizing

$$
\begin{aligned}
\min _{\delta f \in \mathscr{D}\left((-\Delta+1)^{\gamma}\right)} J_{\mu}(f)= & \left\|\left(\mathscr{F}_{f}^{\prime} f^{*}\right) \delta f-\left(h^{\delta}-\mathscr{F} f^{*}\right)\right\|_{L^{2}(\Gamma \times(0, T))}^{2} \\
& +\mu\|\delta f\|_{\mathscr{D}\left((-\Delta+1)^{\gamma}\right)}^{2}
\end{aligned}
$$

where $\delta f=f-f^{*}$.

Now, we consider the discretization of the minimization problem. We define an admissible set of unknown source $f$ $(x)$. Suppose that $\left\{\varphi_{s}(x), s=1,2, \cdots, \infty\right\}$ is a basis in $\mathscr{D}($ $\left.(-\Delta+1)^{\gamma}\right)$, let

$$
\begin{gathered}
f(x) \approx \widehat{f}(x)=\sum_{s=1}^{s} a_{s} \varphi_{s}(x), \\
f^{*}(x) \approx \widehat{f^{*}}(x)=\sum_{s=1}^{s} a_{s}^{*} \varphi_{s}(x),
\end{gathered}
$$

where $\widehat{f}$ is the $S$-dimensional approximation solution to $f$ and $S \in \mathbb{N}$ is a truncated level of $f$, and $a_{s}, s=1,2, \cdots, S$ are the expansion coefficients. It is convenient to set a finite dimensional space as

$$
\Phi^{S}=\operatorname{span}\left\{\varphi_{1}, \varphi_{2}, \cdots, \varphi_{S}\right\}
$$




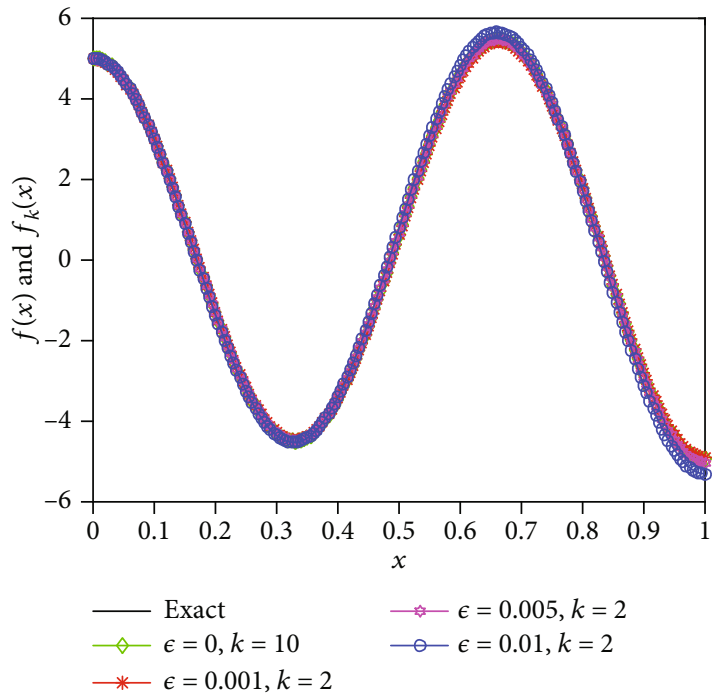

(a) $\alpha=(0.9,0.3,0.1)$

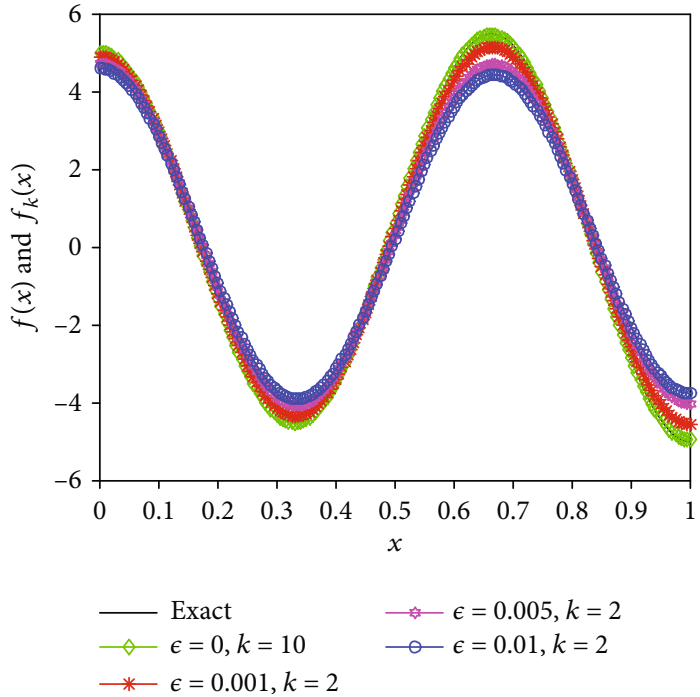

(b) $\alpha=(0.7,0.6,0.2)$

FIGURE 1: The numerical results for Example 16 for various noise levels with $\mu=10^{-6} \delta^{3 / 4}$ and $\gamma=0$.

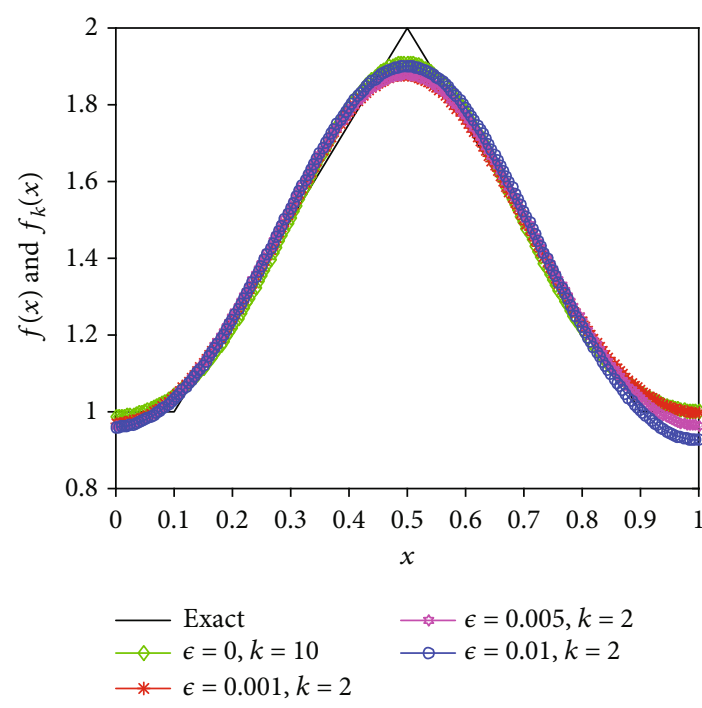

(a) $\alpha=(0.9,0.6,0.1)$

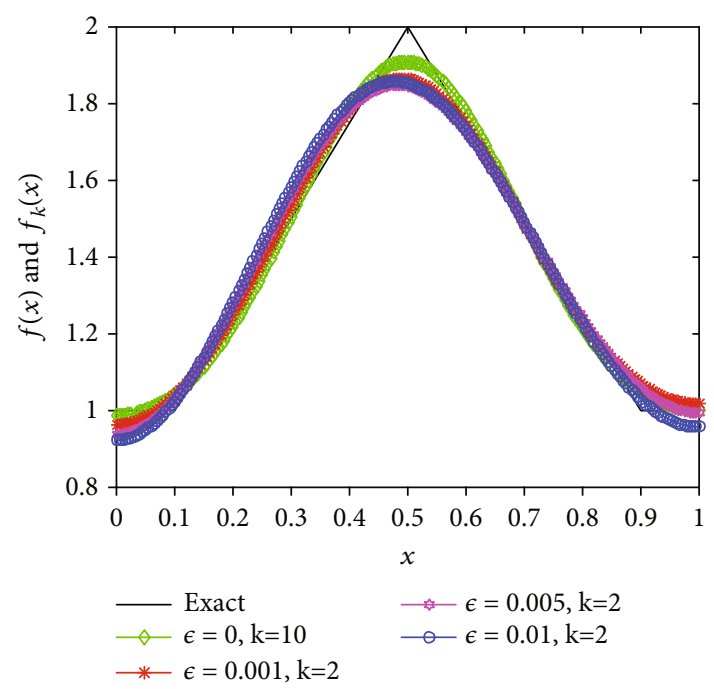

(b) $\alpha=(0.8,0.7,0.6)$

FIgURE 2: The numerical results for Example 17 for various noise levels with $\mu=10^{-5} \delta^{3 / 4}$ and $\gamma=1 / 10$.

TABLE 1: The relative error $\mathrm{re}_{k}$ with different noise levels $\epsilon$ and fractional orders $\alpha$ for Example 16.

\begin{tabular}{lcccc}
\hline $\begin{array}{l}\alpha \\
\mathrm{re}_{k} \\
\epsilon\end{array}$ & 0 & 0.001 & 0.005 & 0.01 \\
\hline$(0.9,0.3,0.2)$ & 0.0059 & 0.0135 & 0.0126 & 0.0484 \\
$(0.8,0.7,0.6)$ & 0.0060 & 0.0082 & 0.0357 & 0.0941 \\
$(0.7,0.5,0.2)$ & 0.0059 & 0.0900 & 0.2258 & 0.3104 \\
$(0.6,0.3,0.2)$ & 0.0059 & 0.1217 & 0.6046 & 0.7569 \\
$(0.5,0.3,0.2)$ & 0.0058 & 0.2947 & 0.7656 & 0.8595 \\
\hline
\end{tabular}

and $S$-dimensional vector $\mathbf{a}=\left(a_{1}, a_{2}, \cdots, a_{S}\right) \in \mathbb{R}^{S}$. We identify an approximation $\widehat{f}(x) \in \Phi^{S}$ with a vector $\mathbf{a} \in \mathbb{R}^{S}$.

Based on the above discussions, by setting $u(x, t ; \mathbf{a})=u$ $(x, t ; \widehat{f})$ as a unique solution of the forward problem, a feasible way for numerical solution to solve the following minimization problem

$\min _{\delta \mathbf{a} \in \mathbb{R}^{S}}\left\{\left\|\delta \mathbf{a} \nabla_{\mathbf{a}}^{T} u\left(x, t ; \mathbf{a}^{*}\right)-\left(h^{\delta}-u\left(x, t ; \mathbf{a}^{*}\right)\right)\right\|_{L^{2}(\Gamma \times(0, T))}^{2}+\mu \delta \mathbf{a} A \delta \mathbf{a}^{T}\right\}$,

where $A=\left(\left(\lambda_{i}^{\gamma} \varphi_{i}, \lambda_{j}^{\gamma} \varphi_{j}\right)_{L^{2}(\Omega)}\right) S \times S, \mathbf{a}^{*}=\left(a_{1}^{*}, a_{2}^{*}, \cdots, a_{S}^{*}\right), \delta \mathbf{a}=$ $\mathbf{a}-\mathbf{a}^{*}$, and $\mathbf{a}^{T}$ denotes the transpose of $\mathbf{a}$. 
TABLE 2: The relative error $\mathrm{re}_{k}$ with different noise levels $\epsilon$ and index of penalty term $\gamma$ for $\alpha=(0.8,0.6,0.1)$ in Example 16 .

\begin{tabular}{lcccc}
\hline$\gamma$ & & & & \\
$\mathrm{re}_{k}$ & 0 & 0.001 & 0.005 & 0.01 \\
$\epsilon$ & & & & \\
\hline 0 & 0.0059 & 0.0257 & 0.0348 & 0.0297 \\
$1 / 10$ & 0.0059 & 0.0101 & 0.1851 & 0.2344 \\
$1 / 3$ & 0.0059 & 0.0361 & 0.2462 & 0.4812 \\
$1 / 2$ & 0.0059 & 0.0939 & 0.4363 & 0.5727 \\
\hline
\end{tabular}

TABLE 3: The relative error $\mathrm{re}_{k}$ with different noise levels $\epsilon$ and index of penalty term $\gamma$ for $\alpha=(0.9,0.5,0.2)$ in Example 17 .

\begin{tabular}{lcccc}
\hline $\begin{array}{l}\alpha \\
\mathrm{re}_{k}\end{array}$ & 0 & 0.001 & 0.005 & 0.01 \\
$\epsilon$ & & & & \\
\hline$-1 / 10$ & 0.0194 & 0.0695 & 0.1372 & 0.1889 \\
0 & 0.0194 & 0.0274 & 0.0532 & 0.0801 \\
$-1 / 10$ & 0.0194 & 0.0231 & 0.0261 & 0.0272 \\
$1 / 4$ & 0.0194 & 0.0238 & 0.0687 & 0.0704 \\
$1 / 2$ & 0.0194 & 0.0333 & 0.0760 & 0.0772 \\
\hline
\end{tabular}

Next, we give an inversion algorithm for determining coefficient a. Given the initial $\mathbf{a}^{0}$ and let $\mathbf{a}^{*}=\mathbf{a}^{0}$. Suppose $\mathbf{a}^{k}$ $\in \mathbb{R}^{S}$ is the $k$ th step iteration value, then we obtain $(k+1$ )th step approximation by solving

$$
\mathbf{a}^{k+1}=\mathbf{a}^{k}+\delta \mathbf{a}^{k}, k=0,1, \cdots,
$$

where $\delta \mathbf{a}^{k}$ denotes a perturbation of $\mathbf{a}^{k}$ for each $k$ and $k$ is the number of iterations. For convenience of writing, $\mathbf{a}^{k}$ and $\delta \mathbf{a}^{k}$ are also abbreviated as a and $\delta \mathbf{a}$, respectively. know,

Now, we compute $\delta \mathbf{a}$ by the variational theory. As we

$$
\begin{aligned}
& \nabla_{\mathbf{a}} u\left(x, t ; \mathbf{a}^{k}\right) \\
& \quad=\left(\frac{u\left(x, t ; \mathbf{a}^{k}+\tau \mathbf{e}_{1}\right)-u\left(x, t ; \mathbf{a}^{k}\right)}{\tau}, \cdots, \frac{u\left(x, t ; \mathbf{a}^{k}+\tau \mathbf{e}_{S}\right)-u\left(x, t ; \mathbf{a}^{k}\right)}{\tau}\right),
\end{aligned}
$$

where $\mathbf{e}_{i}=(0, \cdots, 0,1,0, \cdots, 0), i=1, \cdots, S$. Since operator $\mathscr{F}$ is linear respect to the source $f$, we have

$$
\nabla_{\mathbf{a}} u\left(x, t ; \mathbf{a}^{k}\right)=\left(u\left(x, t ; \mathbf{e}_{1}\right), \cdots, u\left(x, t ; \mathbf{e}_{S}\right)\right)=:\left(b_{1}, \cdots, b_{S}\right) .
$$

By the variational theory, problem (64) is equivalent to the following normal equations

$$
(\mu A+B) \delta \mathbf{a}^{T}=V,
$$

where $B=\left(\left(b_{1}, b_{j}\right)_{L^{2}(\Gamma \times(0, T))}\right) S \times S$ and $V=\left(\left(h^{\delta}(x, t)-u(x\right.\right.$, $\left.\left.t ; \mathbf{a}^{k}\right), b_{i}\right)_{L^{2}(\Gamma \times(0, T))} S \times 1$.

\section{Numerical Experiments}

In this section, we present the numerical results for three examples in one-dimensional and two-dimensional cases to show the effectiveness of the Levenberg-Marquardt method.

The noisy data is generated by adding a random perturbation, i.e.,

$$
h^{\delta}=h+\epsilon h \cdot(2 \cdot \operatorname{rand}(\operatorname{size}(h))-1) .
$$

The corresponding noise level is calculated by $\delta=$ $\left\|h^{\delta}-h\right\|_{L^{2}\left(0, T ; L^{2}(\Gamma) \times(0, z t)\right)}$.

To show the accuracy of numerical solution, we compute the approximate $L^{2}$ error denoted by

$$
r e_{k}=\frac{\left\|f_{k}(x)-f(x)\right\|_{L^{2}(\Omega)}}{\left\|f_{k}(x)\right\|_{L^{2}(\Omega)}},
$$

where $f_{k}(x)$ is the coefficient term reconstructed at the $k$ th iteration, and $f(x)$ is the exact solution.

The residual $E_{k}$ at the $k$ th iteration is given by

$$
E_{k}=\left\|u\left(x, t ; f_{k}\right)-h^{\delta}(x, t)\right\|_{L^{2}(\Gamma \times(0, T))} .
$$

In an iteration algorithm, the most important work is to find a suitable stopping rule. In this study, we use the wellknown discrepancy principle [33], i.e., we choose $k$ satisfying the following inequality

$$
E_{k} \leq \zeta \delta<E_{k-1},
$$

where $\zeta>1$ is a constant and can be taken heuristically to be 1.01, as suggested by Hanke and Hansen [34].

6.1. One-Dimensional Case. Without loss of generality, we set $m=3, q_{j}=1(j=1,2,3)$, and $\Omega_{T}=(0,1) \times(0, T)$. The grid points on $[0,1]$ and $[0, T]$ are both 201 when solving the direct problem by finite difference method in [35]. In this case, we take $\Gamma$ as a point $x=0$ and $\Phi_{S}$ is chosen as a subspace of eigenfunctions

$$
\Phi_{S}=\operatorname{span}\{1, \sqrt{2} \cos (\pi x), \cdots, \sqrt{2} \cos ((S-1) \pi x)\} .
$$

Example 16. We firstly test a smooth solution. Take an unknown source $f(x)=5 \cos (3 \pi x)+10 x^{2}(1-x)^{2}$, the temporal source $r(t)=\exp (t)$, the initial $\phi(x)=\cos (\pi x)$, and the boundary $b(0, t)=1+t, b(1, t)=-1+t$. The boundary data $u(0, t)$ is obtained by solving the direct problem (1) by using the finite difference method. The numerical results for Example 16 by using the discrepancy principle for various noise levels in the cases of $\alpha=(0.9,0.3,0,1)$ and $\alpha=($ $0.7,0.6,0.2)$ are shown in Figures $1(\mathrm{a})$ and $1(\mathrm{~b})$, respectively. 


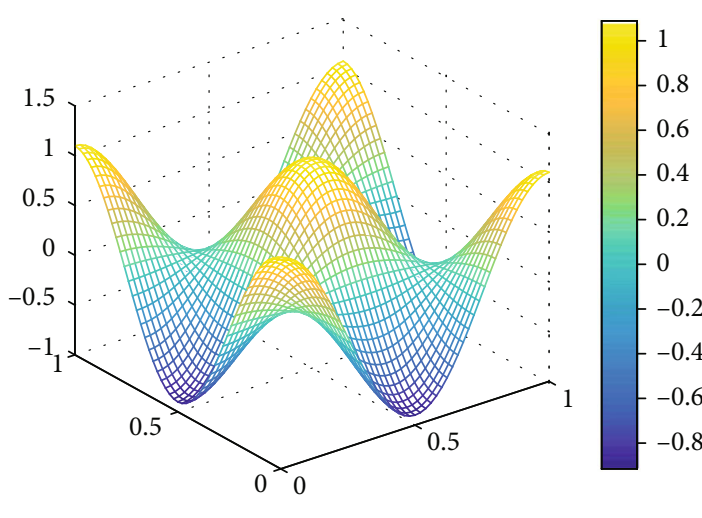

Exact

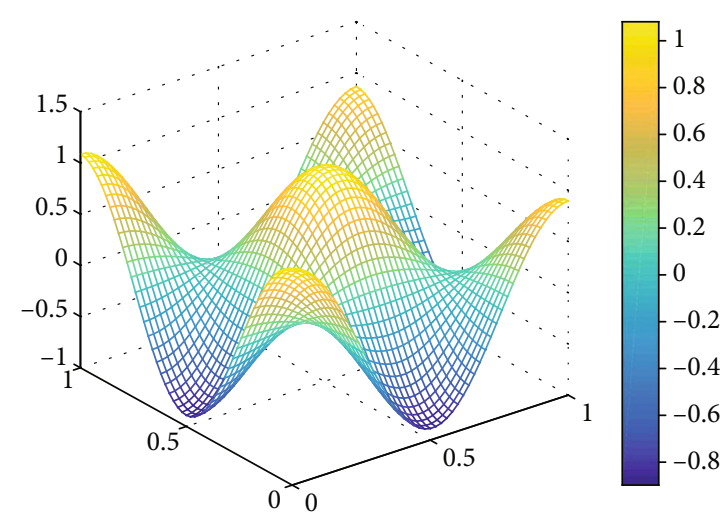

Numerical

(a) Exact solution

(b) Regularized solution

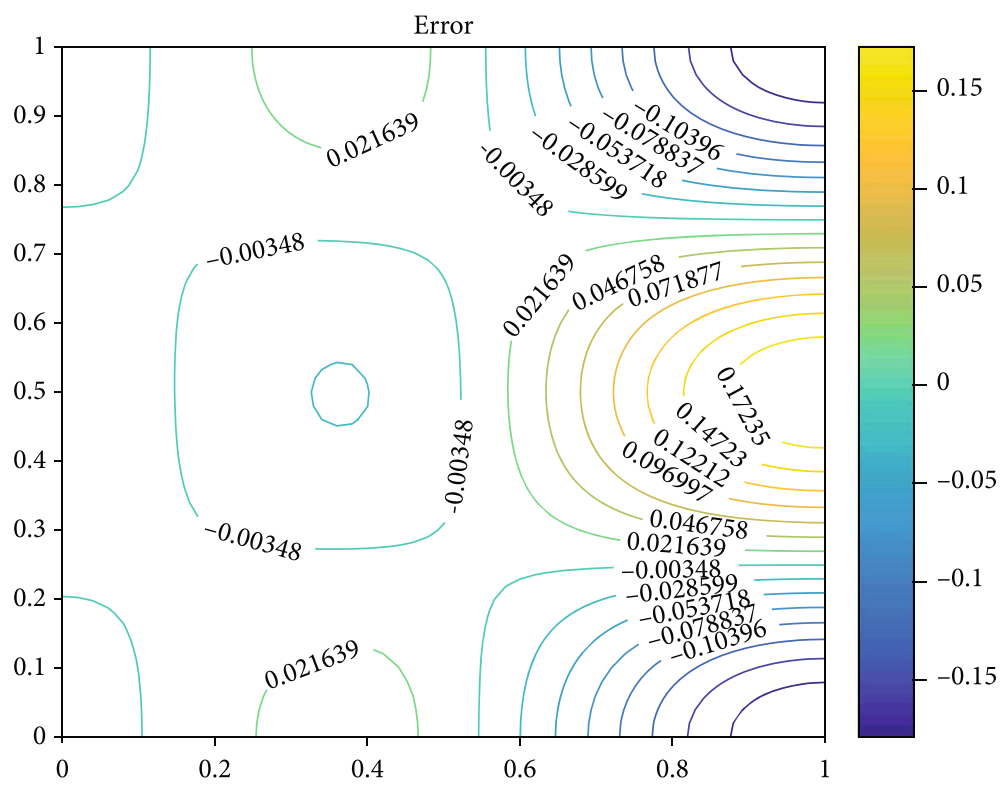

(c) Absolute error

Figure 3: The numerical results for Example 18 for $\alpha=(0.9,0.5,0.1)$ with $\mu=0.01 \delta^{3 / 4}$.

We choose the initial guess as $f_{0}=f^{*}=0$, the truncated level $S=4$.

Example 17. In the second example, we test a nonsmooth solution with a cusp. Take an unknown source

$$
f(x)= \begin{cases}1, \quad 0 \leq x<0.1 & \\ 1+2.5(x-0.1), & 0.5 \leq x<0.5 \\ 2+2.5(0.5-x), & 0.5 \leq x<0.9 \\ 1, \quad 0.9 \leq x \leq 1, & \end{cases}
$$

and the rest of definite conditions are the same as Example 16. The boundary data $u(0, t)$ is obtained by solving the direct problem (1) by using the finite difference method. The numerical results for Example 17 by using the discrepancy principle for various noise levels in the cases of $\alpha=$ $(0.9,0.6,0,1)$ and $\alpha=(0.8,0.7,0.6)$ are shown in Figures $2(a)$ and 2 (b), respectively. We choose the initial guess as $f_{0}=0$ and $f^{*}=0$, the truncated level $S=5$.

From Tables 1 and 2, we can come to the following conclusions. First, it is observed that the dominate fractional order $\alpha_{1}$ has a greater influence on the accuracy of the numerical inversion results. For each fixed $\epsilon$, we can see that the numerical results become worse as the dominate fractional order $\alpha_{1}$ becomes smaller, but it is not sensitive to the rest of the orders $\alpha_{2}$ or $\alpha_{3}$. On the other hand, the numerical results become worse as the index of penalty term $\gamma$ becomes larger for each fixed $\epsilon$. Since here we test a smooth function, the penalty term with high index may not have positive effect to the numerical inversion. So we choose $\gamma=0$ in the first example.

From Table 3, we can see that the regularity of the penalty term can do modify the computation result well for a nonsmooth numerical test. But this regularity should not be too strong, or it will have the opposite effect. We find that $\gamma=1$ $/ 10$ is a better index by the numerical test, so we choose $\gamma$ $=1 / 10$ in the second example. 


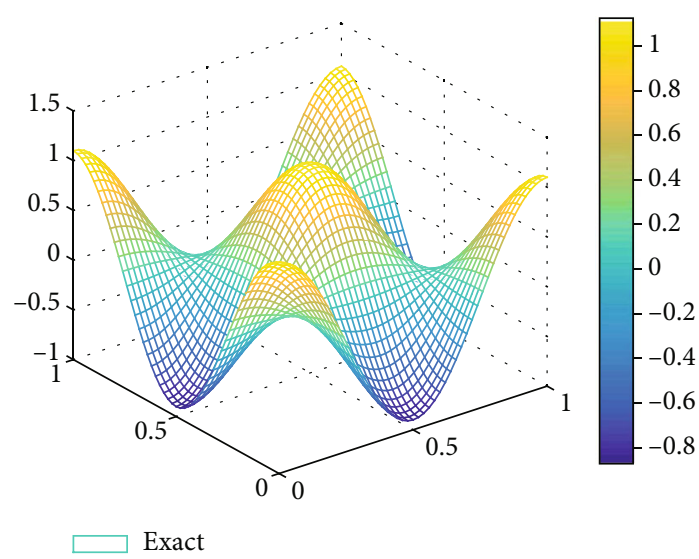

(a) Exact solution

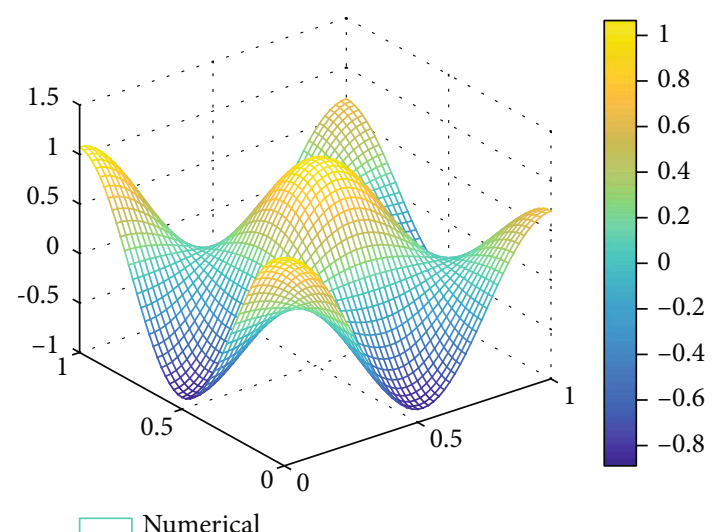

(b) Regularized solution

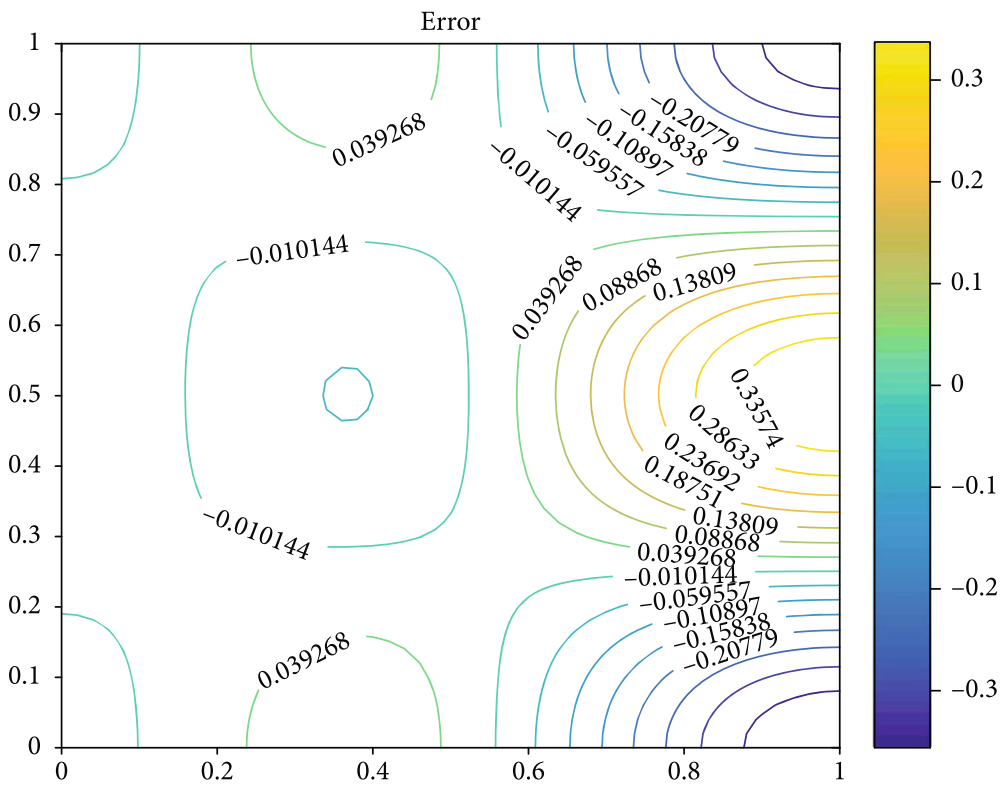

(c) Absolute error

FIGURE 4: The numerical results for Example 18 for $\alpha=(0.6,0.5,0.4)$ with $\mu=0.01 \delta^{3 / 4}$.

6.2. Two-Dimensional Case. In a two-dimensional case, we present one numerical example to show the effectiveness of our method. We also assume $T=1, m=3, q_{j}=1(j=1,2,3)$ without loss of generality. The space domain $\Omega$ is taken as ( $0,1) \times(0,1)$. The grid points on $[0,1]$ and $[0, T]$ are both 51 when solving the direct problem by finite difference method in [35]. We take $\Gamma=\{(x, y) \mid x=0,0 \leq y \leq 1\}$. In this case, we take $\Phi^{S_{1} \times S_{2}}=\operatorname{span}\left\{\varphi_{i}(x) \varphi_{j}(y)\right\}$, where

$$
\begin{aligned}
\varphi_{i}(x) & = \begin{cases}1, \quad i=1, & i=1,2, \cdots, S_{1}, \\
\sqrt{2} \cos ((i-1) \pi x), & i>1,\end{cases} \\
\varphi_{j}(y) & = \begin{cases}1, \quad j=1, & j=1,2, \cdots, S_{2} . \\
\sqrt{2} \cos ((i-1) \pi y), & j>1,\end{cases}
\end{aligned}
$$

Example 18. Assume $f(x, y)=\cos (2 \pi x) \cos (2 \pi y)+1 / 10$ $\exp \left(-1 / 3\left(x^{3}-3 x^{2}\right)\left(2 y^{3}-3 y^{2}\right)\right)$ and $r(t)=\exp (t), \phi(x, y)$
$=0, b=0$. The boundary data $u(0, y, t)$ is obtained by solving direct problem (1). The exact source solutions, reconstructed source terms, and the absolute errors between the exact source functions and the numerical solutions for $\alpha=($ $0.9,0.5,0,1)$ and $\alpha=(0.6,0.5,0,4)$ are shown in Figures 3 and 4 by taking noise level $\epsilon=0.01$ and the regularization parameter $\mu=0.01 \delta^{3 / 4}$, respectively. Here, we choose the initial guess as $f_{0}=0$ and $f^{*}=0$. The truncated levels are $S_{1}=$ $S_{2}=3$.

From Figures 3 and 4, we can see the proposed method still yields relatively accurate numerical solutions for a twodimensional example.

Remark 19. As we know, we need to compute the gradient of an abstract operator by the Levenberg-Marquardt method, and we usually use numerical differential method to approximately substitute the gradient when it is hard to obtain the exact expression for the gradient. So, we need to introduce a numerical differentiation step $\tau$. However, a lot of 
numerical examples are sensitive to $\tau$. In this computation, we have successfully avoided the problem of selection of the differential step. This is because that the operator $\mathscr{F}$ is linear to $f$ and the gradient in (66) can be replaced by (67) where the gradient is not dependent to $\tau$. But when we calculate (66) by a finite difference, the initial and boundary conditions need to be homogeneous.

\section{Conclusions}

In this paper, we investigate an inverse space-dependent source problem of a multiterm time-fractional diffusion equation with nonhomogeneous boundary condition in a general domain. The existence and uniqueness of a weak solution for the direct problem are obtained by the LaxMilgram theorem. Then, the uniqueness for the inverse problem is provided by using the analytic continuation and the Laplace transformation. Finally, we employ the LevenbergMarquardt regularization method to find an approximation of the source function.

In fact, we have obtained the well-posedness of the direct problem and also proved the uniqueness of the inverse problem for the case of nonhomogeneous boundary in the present paper. But there are many other important problems still remain open. For theoretical aspect, the conditional stability of the inverse source problem is still not obtained. For numerical computation, on the one hand, we have indeed improved the original M-L algorithm. However, some nonsmooth source functions, e.g., discontinuous function, are not reconstructed well although the uniqueness holds true theoretically.

\section{Data Availability}

The data that support the findings of this study are available from the corresponding author (Liangliang Sun, Email: sunl10321@163.com), upon reasonable request.

\section{Conflicts of Interest}

The authors declare that there are no conflicts of interest regarding the publication of this article.

\section{Acknowledgments}

This work is supported by the Innovation Capacity Improvement Project for Colleges and Universities of Gansu Province (no. 2020B-088), the Young Teachers' Scientific Research Ability Promotion Project of NWNU (no. NWNU-LKQN18-31), and the Doctoral Scientific Research Foundation of NWNU (no. 6014/0002020204).

\section{References}

[1] Y. Luchko, "Initial-boundary-value problems for the generalized multi-term time-fractional diffusion equation," Journal of Mathematical Analysis and Applications, vol. 374, no. 2, pp. 538-548, 2011.

[2] Y. Liu, "Strong maximum principle for multi-term timefractional diffusion equations and its application to an inverse source problem," Computers \& Mathematcs with Applications, vol. 73, no. 1, pp. 96-108, 2017.

[3] Z. Li, Y. Liu, and M. Yamamoto, "Initial-boundary value problems for multi-term time-fractional diffusion equations with positive constant coefficients," Applied Mathematics and Computation, vol. 257, pp. 381-397, 2015.

[4] M. Zheng, F. Liu, V. Anh, and I. Turner, "A high-order spectral method for the multi-term time-fractional diffusion equations," Applied Mathematical Modelling, vol. 40, no. 7-8, pp. 4970-4985, 2016.

[5] B. Jin, R. Lazarov, Y. Liu, and Z. Zhou, "The Galerkin finite element method for a multi-term time-fractional diffusion equation," Journal of Computational Physics, vol. 281, pp. 825-843, 2015.

[6] W. Wang, M. Yamamoto, and B. Han, "Numerical method in reproducing kernel space for an inverse source problem for the fractional diffusion equation," Inverse Problems, vol. 29, no. 9, p. 095009, 2013.

[7] Z. Q. Zhang and T. Wei, "Identifying an unknown source in time-fractional diffusion equation by a truncation method," Applied Mathematics and Computation, vol. 219, no. 11, pp. 5972-5983, 2013.

[8] J.-G. Wang, Y.-B. Zhou, and T. Wei, "Two regularization methods to identify a space-dependent source for the timefractional diffusion equation," Applied Numerical Mathematics, vol. 68, pp. 39-57, 2013.

[9] T. Wei and J.-G. Wang, "A modified quasi-boundary value method for the backward time-fractional diffusion problem," ESAIM: Mathematical Modelling and Numerical Analysis, vol. 48, no. 2, pp. 603-621, 2014.

[10] J.-G. Wang and T. Wei, "Quasi-reversibility method to identify a space-dependent source for the time-fractional diffusion equation," Applied Mathematical Modelling, vol. 39, no. 20, pp. 6139-6149, 2015.

[11] Y. Zhang and X. Xu, "Inverse source problem for a fractional diffusion equation," Inverse Problems, vol. 27, no. 3, article 035010, 2011.

[12] T. Wei, L. Sun, and Y. Li, "Uniqueness for an inverse spacedependent source term in a multi-dimensional timefractional diffusion equation," Applied Mathematics Letters, vol. 61, pp. 108-113, 2016.

[13] X. B. Yan and T. Wei, "Determine a space-dependent source term in a time fractional diffusion-wave equation," Acta Applicandae Mathematicae, vol. 165, no. 1, pp. 163-181, 2020.

[14] D. Jiang, Z. Li, Y. Liu, and M. Yamamoto, "Weak unique continuation property and a related inverse source problem for time-fractional diffusion-advection equations," Inverse Problems, vol. 33, no. 5, p. 055013, 2017.

[15] Y. Li, L. Sun, Z. Zhang, and T. Wei, "Identification of the timedependent source term in a multi-term timefractional diffusion equation," Numerical Algorithms, vol. 82, no. 4, pp. 1279-1301, 2019.

[16] A. Kilbas, H. Srivastava, and J. Trujillo, Theory and Applications of Fractional Differential Equations, Elsevier, Amsterdam, 2006.

[17] I. Podlubny, Fractional Differential Equations, Academic Press, San Diego, 1999.

[18] Y. Luchko and R. Gorenflo, "An operational method for solving fractional differential equations with the Caputo derivatives," Acta Mathematica Vietnamica, vol. 24, no. 2, pp. 207233, 1999. 
[19] R. A. Adams, Sobolev Spaces, and Academic Press, Pure and Applied Mathematics, vol. 65, A subsidiary of Harcourt Brace Jovanovich, Publishers, New York-London, 1975.

[20] X. Li and C. Xu, "A space-time spectral method for the time fractional diffusion equation," SIAM Journal on Numerical Analysis, vol. 47, no. 3, pp. 2108-2131, 2009.

[21] T. Wei and J. G. Wang, "Determination of robin coefficient in a fractional diffusion problem," Applied Mathematical Modelling, vol. 40, no. 17-18, pp. 7948-7961, 2016.

[22] L. Sun and T. Wei, "Identification of the zeroth-order coefficient in a time fractional diffusion equation," Applied Numerical Mathematics, vol. 111, pp. 160-180, 2017.

[23] R. Courant and D. Hilbert, Methods of Mathematical Physics, vol. I, Interscience Publishers, Inc., New York, N.Y., 1953.

[24] V. Isakov, "Inverse Problems for Partial Differential Equations," in Applied Mathematical Sciences, vol. 127, SpringerVerlag, New York, 2006.

[25] J. L. Lions and E. Magenes, Non-homogeneous Boundary Value Problems and Applications, Springer-Verlag, New York, 1972.

[26] H. Brezis, Functional Analysis, Sobolev Spaces and Partial Differential Equations, Springer, New York, 2011.

[27] A. Kirsch, "An Introduction to the Mathematical Theory of Inverse Problems," in Applied Mathematical Sciences, vol. 120, Springer-Verlag, New York, 1996.

[28] K. Levenberg, "A method for the solution of certain non-linear problems in least squares," Quarterly of Applied Mathematics, vol. 2, no. 2, pp. 164-168, 1944.

[29] D. W. Marquardt, "An algorithm for least-squares estimation of nonlinear parameters," Journal of the Society for Industrial and Applied Mathematics, vol. 11, no. 2, pp. 431-441, 1963.

[30] L. Sun, X. Yan, and T. Wei, "Identification of time-dependent convection coefficient in a time-fractional diffusion equation," Journal of Computational and Applied Mathematics, vol. 346, pp. 505-517, 2019.

[31] L. Sun, Y. Zhang, and T. Wei, "Recovering the time-dependent potential function in a multi-term time-fractional diffusion equation," Applied Numerical Mathematics, vol. 135, pp. 228-245, 2019.

[32] K. Liao and T. Wei, "Identifying a fractional order and a space source term in a time-fractional diffusion-wave equation simultaneously," Inverse Problems, vol. 35, no. 11, p. 115002, 2019.

[33] V. A. Morozov, Methods for Solving Incorrectly Posed Problems, Springer-Verlag, New York, 1984.

[34] M. Hanke and P. Hansen, "Regularization methods for largescale problems," Survey. Math. Indust., vol. 3, no. 4, pp. 253315, 1993.

[35] D. A. Murio, "Implicit finite difference approximation for time fractional diffusion equations," Computers \& Mathematcs with Applications, vol. 56, no. 4, pp. 1138-1145, 2008. 